\title{
Test Facility Location
}

National Cancer Institute

\section{Source}

National Cancer Institute. Test Facility Location. NCI Thesaurus. Code C90468.

The geographic area in which a test is conducted. 